\title{
TECNOLOGIAS EM SAÚDE PARA A PROMOÇÃO DO AUTOCUIDADO EM PACIENTES COM HANSENÍASE: EXPLORANDO EVIDÊNCIAS CIENTÍFICAS
}

\author{
HEALTH TECHNOLOGIES FOR SELF-CARE \\ PROMOTION IN PATIENTS WITH LEPROSY: \\ EXPLORING SCIENTIFIC EVIDENCE
}

\section{TECNOLOGÍAS EN SALUD PARA LA PROMOCIÓN DE AUTOCUIDADO EN PACIENTES CON LEPRA: EXPLORANDO LA EVIDENCIA CIENTÍFICA}

\author{
Jeane Lima Cavalcante ${ }^{1}$ \\ Samyra Paula Lustoza Xavier ${ }^{2}$ \\ Jennifer Ferreira Figueiredo Cabral ${ }^{3}$ \\ Maria Corina Amaral Viana ${ }^{4}$ \\ Edilma Gomes Rocha Cavalcante
}

Como citar este artigo: Cavalcante JL, Xavier SPL, Cabral JFF, Viana MCA, Cavalcante EGR. Tecnologias em saúde para a promoção do autocuidado em pacientes com hanseníase: explorando evidências científicas. Rev baiana enferm. 2019;33:e33369.

\begin{abstract}
Objetivo: identificar as principais tecnologias em saúde aplicadas às pessoas com hanseníase, para a promoção do autocuidado. Método: revisão integrativa realizada nas bases de dados Hansen, LILACS, BDENF, MEDLINE, SciELO, CINAHL, Web of Science e na Biblioteca Virtual em Saúde Hanseníase, por meio do cruzamento dos descritores Hanseníase, Tecnologia em Saúde e Autocuidado. Foram incluídos os artigos publicados no período de 2008 a 2018 nos idiomas inglês, espanhol e português. Resultados: foram selecionados 19 artigos, nos quais se identificaram as tecnologias educativas, com base na formação de grupos, palestras e oficinas, assim como as tecnologias assistenciais, com base na observação, investigação sobre as práticas de cuidados e na utilização de instrumentos. Conclusão: as tecnologias assistenciais foram as mais aplicadas às pessoas com hanseníase, para a promoção do autocuidado.
\end{abstract}

Descritores: Hanseníase. Tecnologia em Saúde. Autocuidado.

Objective: to identify the main health technologies applied to people with leprosy for self-care promotion. Method: integrative review performed at Hansen, LILACS, BDENF, MEDLINE, SCiELO, CINAHL, Web of Science and in the Virtual Health Library Leprosy databases, through the intersection of the descriptors Leprosy, Health Technology and

\footnotetext{
Enfermeira. Especialista em Saúde da Família. Universidade Regional do Cariri. Crato, Ceará, Brasil. jeanecavalcante2009@hotmail.com. https://orcid.org/0000$0001-9074-8263$.

Enfermeira. Mestre em Enfermagem. Docente da Universidade Regional do Cariri. Iguatu, Ceará, Brasil. https://orcid.org/0000-0002-5295-7627.

Enfermeira. Especialista em Saúde da Família, Dermatologia, Qualidade e Segurança do Paciente, Estomaterapia. Enfermeira do Hospital Regional do Cariri. Juazeiro do Norte, Ceará, Brasil. https://orcid.org/0000-000 I-74I2-3773.

Enfermeira. Mestre em Enfermagem. Doutora em Enfermagem. Professora da Universidade Regional do Cariri. Crato, Ceará, Brasil. http://orcid.org/0000-00026890-9400.

5 Enfermeira. Mestre em Cuidados Clínicos. Doutora em Enfermagem. Professora da Universidade Regional do Cariri. Crato, Ceará, Brasil. https://orcid.org/0000$0002-686 \mid-2383$.
} 
Self-care. The sample included articles published in the period from 2008 to 2018 in English, Spanish and Portuguese. Results: 19 articles were selected, which identified the educational technologies, based on group creation, lectures and workshops, as well as assistive technologies, based on observation, research on care practices and use of instruments. Conclusion: assistive technologies were the most applied to people with leprosy, for self-care promotion.

Descriptors: Leprosy. Health Technology. Self-care.

Objetivo: identificar las principales tecnologías en salud aplicadas a personas con lepra, para la promoción del autocuidado. Método: revisión integradora realizada en las bases de datos Hansen, LILACS, BDENF, MEDLINE, SCiELO, CINAHL, Web of Science y en la Biblioteca Virtual en Salud Lepra, a través de la intersección de los descriptores de Lepra, Tecnología en Salud y Autocuidados. Se incluyeron artículos publicados en el periodo de 2008 a 2018 en inglés, español y portugués. Resultados: se seleccionaron 19 artículos, que identificaron las tecnologías educativas basadas en la formación de grupos, conferencias y talleres, así como las tecnologías de asistencia, basadas en la observación, la investigación sobre las prácticas de cuidado y el uso de instrumentos. Conclusión: las tecnologías de asistencia fueron las más aplicadas a las personas con lepra, para la promoción del autocuidado.

Descriptores: Lepra. Tecnología en Salud. Autocuidado.

\section{Introdução}

A hanseníase é um grave problema de saúde pública mundial, em decorrência da relevância do seu quadro social. No ano de 2012, foram registrados, mundialmente, 232.587 casos novos da doença, dos quais 2.420 casos concentraram-se no continente americano, com 2.234 notificações apenas no Brasil $^{(1)}$. Em 2015, 14 países representaram 95\% da carga global de hanseníase nesse período e o Brasil relatou 26.395, configurando $13 \%$ dos novos casos globais ${ }^{(2)}$. Dados mostram que o Brasil é o segundo país mais endêmico para a doença, depois da Índia ${ }^{(3)}$.

Com vistas a reduzir a carga de hanseníase no mundo, a Organização Mundial da Saúde (OMS) elaborou a Estratégia Global para Hanseníase 2016-2020, cujas ações devem ser realizadas no âmbito global e local, de acordo com três pilares: fortalecimento do controle, coordenação e parceria do governo; combate à hanseníase e às suas complicações; combate à discriminação e promoção da inclusão ${ }^{(2)}$.

Considerando a magnitude da doença e buscando diminuir as problemáticas decorrentes da hanseníase nacionalmente, o Brasil implementou, em 2010, o Programa Nacional de Controle da Hanseníase (PNCH) cujas Ações de Controle na Hanseníase (ACH) estão inseridas na Atenção Primária a Saúde (APS) e são adotadas pela OMS como estratégias para melhor resolubilidade de atenção à saúde e redução da doença ${ }^{(4-5)}$.
O principal objetivo do PNCH é diagnosticar, tratar e curar todos os casos, especialmente o controle da morbidade por meio de medidas preventivas simples e de fácil execução, como a constante busca para a detecção precoce dos casos, fornecimento, acompanhamento e orientação profissional referente ao tratamento com o esquema medicamentoso poliquimioterápico (PQT), além de ações realizadas no intuito de prevenir incapacidades e/ou reabilitar o indivíduo ${ }^{(6)}$. Cabe ainda destacar a produção da saúde no contexto da prática de ensino-aprendizagem e da integralidade da assistência fornecida a pessoa com hanseníase, sendo essas ações necessárias para orientação e motivação do autocuidado ${ }^{(7)}$.

No cuidado prestado a esse público, é imprescindível que os profissionais de saúde desenvolvam uma assistência integral, de modo a diminuir as sequelas provenientes da doença. Nesse contexto, destacam-se as tecnologias em saúde, aqui compreendidas como um conjunto de recursos desenvolvidos com base em conhecimentos científicos e de experiências reais, no intuito de agir como transformador de realidades relacionadas às condições de saúde. Essas dividem-se em três tipos, a saber: tecnologias educacionais (dispositivos para mediação do processo de ensinar e aprender), as tecnologias assistenciais (utilizadas na ação do cuidar, aplicada por 
profissionais nos três níveis de atenção) e as tecnologias gerenciais (aplicadas nas atividades de gestão) $^{(8-9)}$.

Estudos que contemplam ações de promoção da saúde desenvolvidas para prevenção e controle de hanseníase mostram que estas ainda são incipientes e ocorrem sempre em menor frequência quando comparados a outros agravos em saúde ${ }^{(10-11)}$. Tal aspecto, expressa a importância do desenvolvimento de estudos que envolvam essa temática.

Ciente do poder transformador da educação em saúde como estratégia que favorece o autocuidado das pessoas com hanseníase, o presente estudo guiou-se pela seguinte questão: Quais as principais tecnologias em saúde utilizadas para a promoção do autocuidado às pessoas com hanseníase?
O objetivo do estudo é identificar as principais tecnologias em saúde aplicadas a pessoas com hanseníase para a promoção do autocuidado.

\section{Método}

Visando ampliar as discussões sobre o assunto, realizou-se uma revisão integrativa da literatura para resumir as evidências e revelar $\mathrm{O}$ conhecimento corrente sobre um tema ${ }^{(12)}$. Para garantir o rigor metodológico desta revisão, utilizou-se como referencial metodológico as etapas propostas por estudo ${ }^{(13)}$ que apresenta conceitos gerais para esse tipo de revisão.

A construção da pergunta de pesquisa envolveu a estratégia Population, Variables e Outcomes (PVO), que permite organizar os elementos de forma estrutural, conforme Quadro 1, que apresenta os componentes, os respectivos termos Medical Subject Headings (MeSH) e os Descritores em Ciências da Saúde (DeCS).

Quadro 1 - Itens da estratégia de pesquisa, componentes e descritores

\begin{tabular}{|l|l|l|l|}
\hline \multicolumn{1}{|c|}{ Itens da Estratégia } & \multicolumn{1}{|c|}{ Componentes } & \multicolumn{1}{c|}{ Descritores MeSH } & \multicolumn{1}{c|}{ Descritores DeCS } \\
\hline Population & Pessoas com hanseníase & Leprosy / Lepra & Hanseníase \\
\hline Variables & Tecnologia em Saúde & Health Technology & Tecnologia em Saúde \\
\hline Outcomes & Autocuidado & Self Care & Autocuidado \\
\hline
\end{tabular}

Fonte: Elaboração própria.

Os critérios de inclusão elencados foram: estudos sobre o autocuidado de pessoas com hanseníase; texto completo disponível nos idiomas português, inglês ou espanhol. Foram excluídos guias de apoio, manuais/cartilhas, artigos que estavam estruturados no formato de editoriais, revisão de literatura, relato de experiência, teses, dissertações e estudos que não responderam à questão de estudo.

A busca dos artigos foi realizada nos meses de outubro e novembro de 2018, por dois pesquisadores independentes, nas seguintes bases de dados: Literatura Latino-Americana em Ciências da Saúde (LILACS), Base Hansen e Bases de Dados de Enfermagem (BDENF), via Biblioteca Virtual em Saúde Hanseníase, na Medical Literature Analyses and Retrieval System Online (MEDLINE) pela PubMed; no repositório da
Scientific Electronic Library Online (SciELO); na Cumulative Index to Nursing and Allied Health Literature (CINAHL) e na Web of Science, pelo portal de periódico da Coordenação de Aperfeiçoamento de Pessoal de Nível Superior (CAPES). A escolha das referidas bases justifica-se pelo escopo de abrangência e por seu impacto nas produções científicas em saúde. Não se realizou recorte temporal, pois se buscou retratar as tecnologias em saúde aplicadas aos pacientes com hanseníase ao longo dos anos.

A utilização de termos MeSH e DeCS ocorreu em função das bases escolhidas, cujo intuito foi atender criteriosamente ao objetivo e delineamento escolhido para construção deste estudo. Nas bases LILACS, Base Hansen e BDENF, a utilização de DeCS apresentou maior resultado quantitativo de artigos, enquanto nas bases 
MEDLINE, SciELO, CINAHL e Web of Science, os termos MeSH apresentaram melhores resultados.

Para sistematizar as buscas, foram realizados cruzamentos dos termos pré-definidos que compunham a estratégia PVO, com o auxílio dos operadores booleanos AND e OR, sendo estes: PVO = Hanseníase AND educação em saúde
OR tecnologia educacional AND autocuidado; $\mathrm{PV}=$ Hanseníase AND educação em saúde OR tecnologia educacional; $\mathrm{PO}=$ Hanseníase AND autocuidado. Esta mesma sequência foi seguida nas bases que utilizaram os termos $\mathrm{MeSH}$ ou DeCS. Tais cruzamentos obtiveram como resultados os dados apresentados na Tabela 1.

Tabela 1 - Artigos encontrados nas bases de dados LILACS, BDENF, Base Hansen, SciELO, MEDLINE, CINAHL e Web of Science, mediante os cruzamentos dos termos MeSH e DeCS. Crato, Ceará, Brasil - 2018

\begin{tabular}{l|c|c|c|c|c|c|c}
\hline \multirow{2}{*}{ Itens } & \multicolumn{3}{c|}{ DeCS } & \multicolumn{4}{c}{ MeSH } \\
\cline { 2 - 7 } & LILACS & Base Hansen & BDENF & MEDLINE & SciELO & CINAHL & $\begin{array}{c}\text { Web of } \\
\text { Science }\end{array}$ \\
\hline PVO & 263 & 137 & 52 & 379 & 34 & 91 & 90 \\
PV & 263 & 137 & 52 & 379 & 34 & - & - \\
PO & 23 & 19 & 8 & 40 & - & - & - \\
\hline
\end{tabular}

Fonte: Elaboração própria.

PVO = Population, Variables and Outcomes; $P V=$ Population, Variables; $P O=$ Populations, Outcomes.

Ainda durante a busca, foram aplicados os filtros: estar disponível para download de forma gratuita e no formato de artigo. Nesta etapa, obtiveram-se 26 na LILACS, 19 na base Hansen, 8 na BDENF, 65 na MEDLINE, 7 na SciELO, 27 na CINAHL e 77 na Web of Science, totalizando 229 artigos.

A primeira etapa do refinamento deu-se com a leitura de títulos e resumos dos 229 estudos. Destes, foram excluídos aqueles que explicitamente não atendiam ao escopo desta pesquisa, resultando em 64 estudos: 12 na LILACS, 3 na Base Hansen, 4 na BDENF, 28 na MEDLINE, 4 na SciELO, 6 na CINAHL e 1 na Web of Science.
A segunda etapa do refinamento contemplou a leitura dos artigos na íntegra, realizada após a anuência de dois pesquisadores, que resultou em 3 estudos na LILACS, 2 na BDENF, 6 na MEDLINE, 4 na SciELO, 3 na CINAHL e 1 na Web of Science, perfazendo um total de 19 artigos, que compuseram a amostra final. Para facilitar a compreensão acerca do processo de busca e seleção dos artigos, utilizou-se o fluxograma do Preferred Reporting Items for Systematic Reviewand Meta-Analyses (PRISMA) $^{(14)}$, apresentado na Figura 1 a seguir: 
Figura 1 - Fluxograma de seleção dos estudos

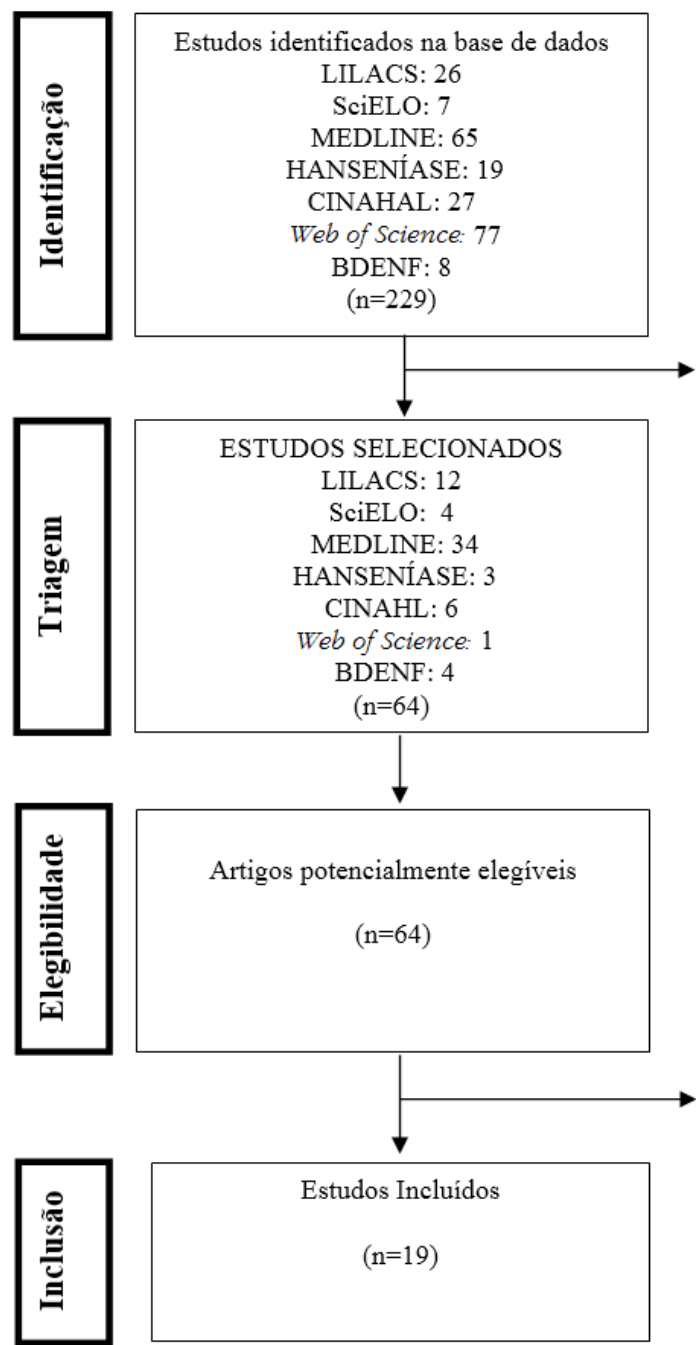

Fonte: Elaboração própria.

A etapa de avaliação dos estudos permitiu identificar, de acordo com a abordagem metodológica e seguindo a pirâmide de evidências exposta em estudo sobre revisão sistemática ${ }^{(15)}$, que 1 artigo estava no nível II, 2 artigos no nível III, 15 artigos no nível IV e 1 artigo no nível V de evidências científicas.

Para extração dos dados, elaborou-se um formulário próprio, contendo dados bibliométricos acerca dosestudosselecionados, tais comoautores, ano, local, tipo de estudo, amostra, ações/tecnologias e resultados, que estão sintetizados na seção a seguir.
Exclusão de estudos:

LILACS: 14 (3 teses, 7 não atenderam ao critério de inclusão e/ou não respondeu à questão do estudo, 3 manuais e 1 relato de experiência).

SciELO: 3 (não atenderam ao critério de inclusão e/ou não respondeu à questão do estudo). MEDLINE: 31 (23 não atenderam aos critérios de inclusão e/ou pergunta do estudo, 5 revisões de literatura, 2 relatos de experiência e 1 editorial. HANSENIASE:16 (5 guias de apoio, 5 manuais/cartilha, 5 teses, 1 não respondeu à questão do estudo).

CINAHL: 21 (não atenderam ao critério de inclusão e/ou não respondeu à questão do estudo).

Web of Science: 76 (não atenderam ao critério de inclusão e/ou não respondeu à questão do estudo). BDENF: 4 (2 não responderam à questão do estudo, 1 relato de experiência e 1 tese).

\section{Exclusão de estudos:}

LILACS: 9 (5 repetidos, 3 teses, 1 relato de experiência).

MEDLINE: 28 ( 5 revisões de literatura, 23 não atenderam ao critério de inclusão e/ou não respondeu à questão do estudo). HANSENÍASE: 3 (do tipo Manuais/cartilhas) CINAHL: 3 (1 repetido e 2 não atenderam aos critérios de inclusão e/ou não respondeu à questão do estudo).

BDENF: 2 (1 relato de experiência, 1 tese).

Os dados referentes ao uso das tecnologias em saúde, bem como às estratégias implementadas, os profissionais envolvidos e resultados obtidos, foram discutidos de forma descritiva, a fim de possibilitar ao leitor melhor compreensão sobre o tema.

\section{Resultados}

Os dados expostos no Quadro 2 sintetizam as principais informações bibliométricas que caracterizam os artigos analisados. 
Quadro 2 - Caracterização dos estudos analisados, segundo autor, local e ano, tipo de estudo e amostra, tecnologias e resultados

(continua)

\begin{tabular}{|c|c|c|c|c|}
\hline Autor & $\begin{array}{l}\text { Local/ } \\
\text { Ano }\end{array}$ & $\begin{array}{l}\text { Tipo de } \\
\text { estudo / } \\
\text { Amostra }\end{array}$ & Tecnologias & Resultados \\
\hline $\begin{array}{l}\text { Lima MCV, } \\
\text { Barbosa FR, } \\
\text { Santos DCM, } \\
\text { Nascimento RD, } \\
\text { D'Azevedo SS }^{(16)}\end{array}$ & $\begin{array}{l}\text { Brasil } \\
2018\end{array}$ & $\begin{array}{l}\text { Estudo } \\
\text { descritivo } \\
24 \text { pessoas }\end{array}$ & $\begin{array}{l}\text { Entrevista } \\
\text { Tecnologia } \\
\text { Assistencial }\end{array}$ & $\begin{array}{l}\text { O estudo apontou que as práticas } \\
\text { de autocuidado realizadas em face, } \\
\text { mãos e pés das pessoas atingidas } \\
\text { pela hanseníase são orientadas e } \\
\text { incentivadas pelos profissionais } \\
\text { de saúde que as acompanham, } \\
\text { porém percebe-se que os desafios } \\
\text { vivenciados pelos entrevistados } \\
\text { estão relacionados a fatores físicos, } \\
\text { ambientais, emocionais e sociais, } \\
\text { como falta de interesse ou de tempo, } \\
\text { baixa renda familiar e dificuldades } \\
\text { devido às incapacidades já instaladas. }\end{array}$ \\
\hline $\begin{array}{l}\text { Pryce J, } \\
\text { Mableson HE, } \\
\text { Choudhary R, } \\
\text { Pandey BD, } \\
\text { Aley D, Betts H, } \\
\text { et al. }\end{array}$ & $\begin{array}{l}\text { Ásia } \\
2018\end{array}$ & $\begin{array}{l}\text { Estudo } \\
\text { descritivo } \\
53 \text { pacientes }\end{array}$ & $\begin{array}{l}\text { Reuniões em } \\
\text { grupos } \\
\text { Tecnologia } \\
\text { Educativa }\end{array}$ & $\begin{array}{l}\text { Em média, os participantes afetados } \\
\text { pela hanseníase demonstraram } 1,8 \text { vezes } \\
\text { mais conhecimento das técnicas de } \\
\text { autocuidado e praticavam } 2,5 \text { vezes mais } \\
\text { frequentemente do que os participantes } \\
\text { afetados por Linfedema Filarial (LF). }\end{array}$ \\
\hline $\begin{array}{l}\text { D'Azevedo SSP, } \\
\text { Freitas EN, } \\
\text { Nascimento LO, } \\
\text { Santos DCM, } \\
\text { Nascimento RD }\end{array}$ & $\begin{array}{l}\text { Brasil } \\
2018\end{array}$ & $\begin{array}{l}\text { Estudo } \\
\text { descritivo } \\
11 \text { pacientes }\end{array}$ & $\begin{array}{l}\begin{array}{l}\text { Reuniões em } \\
\text { grupos }\end{array} \\
\text { Tecnologia } \\
\text { educativa }\end{array}$ & $\begin{array}{l}\text { Redução de incapacidades físicas, } \\
\text { por meio de medidas de prevenção, } \\
\text { educação em saúde, adesão ao } \\
\text { autocuidado e tratamento. Além } \\
\text { disso, esses grupos elevam a } \\
\text { autoestima, proporcionam a } \\
\text { superação de preconceito e } \\
\text { possibilitam o vínculo terapêutico } \\
\text { entre pacientes e profissionais. }\end{array}$ \\
\hline $\begin{array}{l}\text { Morais JR, } \\
\text { Furtado ÉZL }{ }^{(19)}\end{array}$ & $\begin{array}{l}\text { Brasil } \\
2018\end{array}$ & $\begin{array}{l}\text { Estudo } \\
\text { descritivo } \\
73 \text { pacientes }\end{array}$ & $\begin{array}{l}\text { Instrumento } \\
\text { para } \\
\text { avaliação de } \\
\text { incapacidades } \\
\text { Tecnologia } \\
\text { Assistencial }\end{array}$ & $\begin{array}{l}\text { Constatou-se que pacientes do sexo } \\
\text { masculino com baixa escolaridade, } \\
\text { forma clínica dimorfa, virchowiana, } \\
\text { com classificação multibacilar e a } \\
\text { presença de um ou mais nervos } \\
\text { afetados tiveram maior chance } \\
\text { de apresentar algum grau de } \\
\text { incapacidade física, ressaltando a } \\
\text { importância de instrumentos para } \\
\text { avaliar o grau de incapacidade e } \\
\text { assim subsidiar a implementação } \\
\text { de estratégias de prevenção de } \\
\text { incapacidades e, em caso de danos já } \\
\text { existentes, a adoção de medidas que } \\
\text { visassem evitar complicações, por } \\
\text { meio da promoção do autocuidado e } \\
\text { melhoria da qualidade de vida. }\end{array}$ \\
\hline $\begin{array}{l}\text { Moura EGS, } \\
\text { Araújo APM, } \\
\text { Silva MCR, } \\
\text { Cardoso BA, } \\
\text { Holanda MCS, } \\
\text { Conceição AO, } \\
\text { et al. }\end{array}$ & $\begin{array}{l}\text { Brasil } \\
2017\end{array}$ & $\begin{array}{l}\text { Estudo } \\
\text { descritivo } \\
30 \text { pacientes }\end{array}$ & $\begin{array}{l}\text { Instrumento } \\
\text { SALSA/ } \\
\text { avaliar o } \\
\text { autocuidado } \\
\text { Tecnologia } \\
\text { Assistencial }\end{array}$ & $\begin{array}{l}\text { A Escala SALSA é um instrumento de } \\
\text { mensuração da limitação de atividade, } \\
\text { que prioriza o olhar não só para as } \\
\text { deficiências físicas, mas a funcionalidade } \\
\text { do indivíduo. Os domínios da escala } \\
\text { SALSA mostraram dificuldade leve a } \\
\text { moderada em sua grande maioria, com } \\
\text { resultados significantes } \mathrm{p} \leq 0,05 \text {. }\end{array}$ \\
\hline
\end{tabular}


Quadro 2 - Caracterização dos estudos analisados, segundo autor, local e ano, tipo de estudo e amostra, tecnologias e resultados

(continuação)

\begin{tabular}{|c|c|c|c|c|}
\hline Autor & $\begin{array}{c}\text { Local/ } \\
\text { Ano }\end{array}$ & $\begin{array}{c}\text { Tipo de } \\
\text { estudo / } \\
\text { Amostra }\end{array}$ & Tecnologias & Resultados \\
\hline $\begin{array}{l}\text { Maia FB, } \\
\text { Teixeira ER, } \\
\text { Silva GV, } \\
\text { Gomes } \mathrm{MK}^{(21)}\end{array}$ & $\begin{array}{l}\text { Brasil } \\
2016\end{array}$ & $\begin{array}{l}\text { Estudo } \\
\text { descritivo } \\
8 \text { pacientes }\end{array}$ & $\begin{array}{l}\text { Instrumentos } \\
\text { adaptados } \\
\text { para o } \\
\text { autocuidado } \\
\text { Tecnologia } \\
\text { assistencial }\end{array}$ & $\begin{array}{l}\text { A utilização de instrumentos } \\
\text { adaptados para as práticas de } \\
\text { autocuidado rotineiras das pessoas } \\
\text { com hanseníase revelou contribuições } \\
\text { relevantes ao cuidado a esta clientela } \\
\text { referente a sentimentos, percepções e } \\
\text { conteúdos significativos sobre o social, } \\
\text { familiar e dimensões individuais } \\
\text { e também o estigma associado à } \\
\text { hanseníase. }\end{array}$ \\
\hline $\begin{array}{l}\text { Galan NGA, } \\
\text { Beluci ML, } \\
\text { Marciano LHC, } \\
\text { Prado RBR, } \\
\text { Oliveira NGG, } \\
\text { Bonini AG, } \\
\text { et al }\end{array}$ & $\begin{array}{l}\text { Brasil } \\
2015\end{array}$ & $\begin{array}{l}\begin{array}{l}\text { Estudo } \\
\text { descritivo }\end{array} \\
11 \text { pacientes }\end{array}$ & $\begin{array}{l}\text { Observação } \\
\text { Tecnologia } \\
\text { assistencial }\end{array}$ & $\begin{array}{l}\text { Demonstraram dificuldades em } \\
\text { aceitar o comprometimento e } \\
\text { incorporar as práticas diárias por } \\
\text { fatores multicausais. O mesmo } \\
\text { aconteceu com aqueles com grau } 1 .\end{array}$ \\
\hline $\begin{array}{l}\text { Batista TVG, } \\
\text { Vieira CSCA, } \\
\text { Paula } \text { MAB }^{(23)}\end{array}$ & $\begin{array}{l}\text { Brasil } \\
2014\end{array}$ & $\begin{array}{l}\text { Estudo } \\
\text { descritivo } \\
5 \text { mulheres }\end{array}$ & $\begin{array}{l}\text { Oficina de } \\
\text { desenho } \\
\text { Tecnologia } \\
\text { educativa }\end{array}$ & $\begin{array}{l}\text { Os resultados revelaram a não inclusão } \\
\text { ou a desfiguração de mãos e pés. } \\
\text { Existe uma falta de integração dessas } \\
\text { regiões à consciência, acarretando } \\
\text { desestruturação da imagem corporal, } \\
\text { que pode decorrer tanto da perda } \\
\text { de sensibilidade cutânea como da } \\
\text { deformidade advindas da doença. O } \\
\text { reconhecimento da imagem corporal } \\
\text { por meio de desenhos auxilia o } \\
\text { indivíduo a reconhecer sua dor, suas } \\
\text { angústias, esperanças e favorece à } \\
\text { equipe de saúde um olhar integral do } \\
\text { indivíduo com hanseníase. }\end{array}$ \\
\hline $\begin{array}{l}\text { Souza IA, } \\
\text { Ayres JA, } \\
\text { Meneguin S, } \\
\text { Spagnolo RS }\end{array}$ & $\begin{array}{l}\text { Brasil } \\
2014\end{array}$ & $\begin{array}{l}\text { Estudo } \\
\text { descritivo } \\
15 \text { pacientes }\end{array}$ & $\begin{array}{l}\text { Entrevista } \\
\text { sobre o } \\
\text { autocuidado } \\
\text { Tecnologia } \\
\text { assistencial }\end{array}$ & $\begin{array}{l}\text { Ao entrevistar os pacientes sobre } \\
\text { o autocuidado, o estudo deu } \\
\text { visibilidade ao modelo verticalizado, } \\
\text { largamente hegemônico na tradição } \\
\text { das políticas públicas de saúde, } \\
\text { mostrando preocupação em tratar } \\
\text { apenas a doença, desconsiderando } \\
\text { as relações complexas que a envolve. } \\
\text { Reconhecer estas limitações e ter } \\
\text { estratégias para transformá-las em } \\
\text { favor do diálogo entre os membros } \\
\text { da equipe interprofissional são } \\
\text { desafios para avançar as práticas do } \\
\text { autocuidado e do empoderamento } \\
\text { do portador em relação ao } \\
\text { tratamento e à doença. }\end{array}$ \\
\hline
\end{tabular}


Quadro 2 - Caracterização dos estudos analisados, segundo autor, local e ano, tipo de estudo e amostra, tecnologias e resultados

(continuação)

\begin{tabular}{|c|c|c|c|c|}
\hline Autor & $\begin{array}{l}\text { Local/ } \\
\text { Ano }\end{array}$ & $\begin{array}{l}\text { Tipo de } \\
\text { estudo / } \\
\text { Amostra } \\
\end{array}$ & Tecnologias & Resultados \\
\hline $\begin{array}{l}\text { de Vries HJC, } \\
\text { de Groot R, } \\
\text { van Brakel } \mathrm{WH}^{(25)}\end{array}$ & $\begin{array}{l}\text { Amsterdam } \\
2014\end{array}$ & $\begin{array}{l}\text { Estudo } \\
\text { descritivo } \\
72 \text { pacientes }\end{array}$ & $\begin{array}{l}\text { Palestras em } \\
\text { grupos sobre } \\
\text { a prática do } \\
\text { autocuidado } \\
\text { Tecnologia } \\
\text { educativa }\end{array}$ & $\begin{array}{l}\text { O estudo apontou que as palestras } \\
\text { em grupos com pacientes diabéticos } \\
\text { e ex-pacientes de hanseníase para } \\
\text { a prática do autocuidado, apresenta } \\
\text { divergências. O grupo de ex- } \\
\text {-pacientes de hanseníase, apresenta } \\
\text { maior adesão, demonstrando que } \\
\text { deve haver maior interação entres os } \\
\text { pacientes dos dois grupos. Para os } \\
\text { pacientes diabéticos, reconheceram } \\
\text { a comparabilidade com a hanseníase, } \\
\text { devido aos pacientes neuropáticos, } \\
\text { mas apenas } 17 \% \text { demonstraram } \\
\text { interesse em grupos combinados de } \\
\text { autocuidado. }\end{array}$ \\
\hline $\begin{array}{l}\text { Duarte LMCPS, } \\
\text { Simpson CA, } \\
\text { Silva TMS, } \\
\text { Moura IBL, } \\
\text { Isoldi DMR }\end{array}$ & $\begin{array}{l}\text { Brasil } \\
2014\end{array}$ & $\begin{array}{l}\text { Estudo } \\
\text { descritivo } \\
14 \text { pacientes }\end{array}$ & $\begin{array}{l}\text { Grupos para } \\
\text { autocuidado } \\
\text { Tecnologia } \\
\text { educativa }\end{array}$ & $\begin{array}{l}\text { As ações de autocuidado foram } \\
\text { minimamente relatadas pelos } \\
\text { portadores de hanseníase como } \\
\text { positivas, concentrando-se na } \\
\text { lubrificação dos olhos, no uso de } \\
\text { protetor solar, na hidratação da pele } \\
\text { e no uso de calçados. }\end{array}$ \\
\hline $\begin{array}{l}\text { Leite SCC, } \\
\text { Caldeira AP }\end{array}$ & $\begin{array}{l}\text { Brasil } \\
2014\end{array}$ & $\begin{array}{l}\text { Estudo de } \\
\text { intervenção } \\
62 \text { pacientes }\end{array}$ & $\begin{array}{l}\text { Oficinas } \\
\text { itinerantes } \\
\text { Tecnologia } \\
\text { educativa }\end{array}$ & $\begin{array}{l}\text { Observou-se uma redução } \\
\text { significativa dos escores de } \\
\text { sintomas depressivos após a } \\
\text { intervenção proposta }(p<0,001) \text { e } \\
\text { impacto positivo para os domínios } \\
\text { psicológico }(p=0,001) \text {, físico }(p=0,03) \\
\text { e de meio ambiente }(p<0,001) \text {, } \\
\text { mas não para as relações sociais } \\
(p=0,124) \text {. }\end{array}$ \\
\hline $\begin{array}{l}\text { Pinheiro MGC, } \\
\text { Silva SYB, } \\
\text { Silva FS, } \\
\text { Ataide CAV, } \\
\text { Lima IB, } \\
\text { Simpson } \mathrm{CA}^{(28)}\end{array}$ & $\begin{array}{l}\text { Brasil } \\
2014\end{array}$ & $\begin{array}{l}\text { Estudo } \\
\text { descritivo } \\
16 \text { pacientes }\end{array}$ & $\begin{array}{l}\text { Reuniões } \\
\text { educativas e } \\
\text { oficinas } \\
\text { Tecnologia } \\
\text { educativa }\end{array}$ & $\begin{array}{l}\text { Os resultados permitiram a } \\
\text { constatação de que a participação } \\
\text { dos usuários nas reuniões do grupo } \\
\text { contribuiu significativamente para } \\
\text { a aquisição do conhecimento sobre } \\
\text { práticas de autocuidado. }\end{array}$ \\
\hline 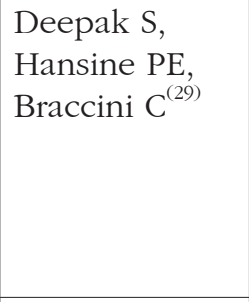 & $\begin{array}{l}\text { Moçambique } \\
2013\end{array}$ & $\begin{array}{l}\text { Estudo } \\
\text { descritivo } \\
299 \text { pacientes }\end{array}$ & $\begin{array}{l}\text { Folhetos } \\
\text { impressos } \\
\text { e materiais } \\
\text { didáticos } \\
\text { Tecnologia } \\
\text { educativa }\end{array}$ & $\begin{array}{l}\text { A maioria dos entrevistados } \\
\text { expressou satisfação sobre sua } \\
\text { participação nos grupos. Além dos } \\
\text { benefícios percebidos em relação } \\
\text { à sua deficiência (redução de } \\
\text { incapacidades). }\end{array}$ \\
\hline $\begin{array}{l}\text { Palmeira IP, } \\
\text { Ferreira MA }\end{array}$ & $\begin{array}{l}\text { Brasil } \\
2012\end{array}$ & $\begin{array}{l}\text { Estudo } \\
\text { descritivo } \\
43 \text { mulheres }\end{array}$ & $\begin{array}{l}\text { Reuniões } \\
\text { individuais } \\
\text { Tecnologia } \\
\text { assistencial }\end{array}$ & $\begin{array}{l}\text { Os resultados mostraram concepções } \\
\text { de corpo relacionadas à saúde (passado) } \\
\text { e à doença (presente), mostrando a } \\
\text { força da estética e da funcionalidade } \\
\text { do corpo, influenciando na participação } \\
\text { e inserção social dessas mulheres. }\end{array}$ \\
\hline
\end{tabular}


Quadro 2 - Caracterização dos estudos analisados, segundo autor, local e ano, tipo de estudo e amostra, tecnologias e resultados

\begin{tabular}{|c|c|c|c|c|}
\hline Autor & $\begin{array}{c}\text { Local/ } \\
\text { Ano }\end{array}$ & $\begin{array}{c}\text { Tipo de } \\
\text { estudo / } \\
\text { Amostra }\end{array}$ & Tecnologias & Resultados \\
\hline Cross $\mathrm{H}^{(31)}$ & $\begin{array}{l}\text { Myanmar } \\
2011\end{array}$ & $\begin{array}{l}\text { Estudo } \\
\text { descritivo } \\
8 \text { pacientes }\end{array}$ & $\begin{array}{l}\text { Grupo focal } \\
\text { Tecnologia } \\
\text { educativa }\end{array}$ & $\begin{array}{l}\text { Os resultados mostraram a } \\
\text { necessidade, bem como a } \\
\text { importância de implementar medidas } \\
\text { de prevenção com o intuito de } \\
\text { minimizar as deformidades/ } \\
\text { incapacidades físicas relacionada } \\
\text { à hanseníase nos programas do } \\
\text { governo. }\end{array}$ \\
\hline $\begin{array}{l}\text { Rodini FCB, } \\
\text { Gonçalves M, } \\
\text { Barros ARSB, } \\
\text { Mazzer N, } \\
\text { Elui VMC, } \\
\text { Fonseca MCR }\end{array}$ & $\begin{array}{l}\text { Brasil } \\
2010\end{array}$ & $\begin{array}{l}\text { Estudo de } \\
\text { intervenção } \\
26 \text { pacientes }\end{array}$ & $\begin{array}{l}\text { Manual de } \\
\text { apoio para o } \\
\text { autocuidado } \\
\text { Tecnologia } \\
\text { educativa }\end{array}$ & $\begin{array}{l}\text { Os nervos mais acometidos } \\
\text { foram o ulnar e o tibial posterior. } \\
\text { Embora o grau de incapacidade } \\
\text { e as deformidades preexistentes } \\
\text { tenham persistido, houve melhora } \\
\text { significativa nos domínios dor e } \\
\text { aspectos sociais do questionário } \\
\text { SF-36, bem como na função } \\
\text { muscular das mãos e dos pés e no } \\
\text { ressecamento da pele. }\end{array}$ \\
\hline $\begin{array}{l}\text { Sathia-Raj Y, } \\
\text { Norman G, } \\
\text { Richard J }\end{array}$ & $\begin{array}{l}\text { Índia } \\
2010\end{array}$ & $\begin{array}{l}\text { Ensaio } \\
\text { Clínico } \\
\text { Randomizado } \\
50 \text { pacientes }\end{array}$ & $\begin{array}{l}\text { Palestras, } \\
\text { prática de } \\
\text { autocuidado } \\
\text { e doação } \\
\text { de sapatos } \\
\text { adequados } \\
\text { Tecnologias } \\
\text { educativa e } \\
\text { assistencial }\end{array}$ & $\begin{array}{l}\text { A educação para o autocuidado } \\
\text { resultou em um nível de } \\
\text { conhecimento e de prática muito } \\
\text { altos em comparação com a área de } \\
\text { controle, chegando até a } 100 \% \text { de } \\
\text { prática em alguns aspectos. }\end{array}$ \\
\hline $\begin{array}{l}\text { Silva Júnior FJG, } \\
\text { Ferreira RD, } \\
\text { Araújo OD, } \\
\text { Camêlo SMA, } \\
\text { Nery IS }{ }^{(34)}\end{array}$ & $\begin{array}{l}\text { Brasil } \\
2008\end{array}$ & $\begin{array}{l}\text { Estudo de } \\
\text { caso } \\
1 \text { paciente }\end{array}$ & $\begin{array}{l}\text { Processo de } \\
\text { Enfermagem } \\
\text { Tecnologia } \\
\text { assistencial }\end{array}$ & $\begin{array}{l}\text { Observou-se que o processo } \\
\text { de enfermagem individualizado } \\
\text { possibilitou à paciente do estudo } \\
\text { ser capaz de dar continuidade ao } \\
\text { tratamento (em relação ao uso dos } \\
\text { medicamentos), haja vista a sua } \\
\text { obstinação, bem como a necessidade } \\
\text { de supervisão das doses diárias } \\
\text { por um familiar, além de realizar } \\
\text { o autocuidado voltado para a } \\
\text { hanseníase de forma culturalmente } \\
\text { satisfatória. }\end{array}$ \\
\hline
\end{tabular}

Fonte: Elaboração própria

Os resultados apontaram que as ações em saúde e tecnologias aplicadas para a promoção do autocuidado em pessoas que convivem com hanseníase transcorreram sobre dois tipos de tecnologias em saúde ${ }^{(8)}$ : educativas e assistenciais.

As tecnologias educativas contemplaram a construção de um manual de autocuidado, cartilhas, folhetos impressos, vídeo, palestras educativas, capacitações e grupos de apoio que enfatizaram a construção de saberes sobre o autocuidado com qualidade, de forma a garantir autonomia e cuidado seguro à clientela ${ }^{(17-18,23,25-29,31-33)}$. Envolveram ainda a percepção, a compreensão e o lúdico como estratégias para promoção do autocuidado. Estas tiveram um impacto positivo no 
tocante à avaliação de sua aplicação, pois facilitaram e ampliaram a compreensão dos pacientes acerca da doença e das práticas para o autocuidado.

Dentre as tecnologias assistenciais, destacaram-se: avaliação periódica da prática do autocuidado e dos fatores intervenientes; promoção da aceitação frente à imagem corporal diante das deformidades físicas decorrentes da hanseníase, uma vez que as ações e reações indicavam os modos de cuidado; aplicação da escala SALSA para avaliação do status físico e funcional de olhos, mãos e pés; reflexões sobre as contribuições da tecnologia assistiva e a abordagem transcultural, bem como a utilização do processo de enfermagem na prestação do cuidado $^{(16,19-22,24,30,33-34)}$.

Quanto às características clínicas dos usuários do serviço de saúde aos quais essas tecnologias foram aplicadas, o maior número de pacientes em tratamento de hanseníase, apresentaram Classificação Operacional Multibacilar e nas formas mais grave da doença (Dimorfa e Virchorwiana), responsáveis pelas incapacidades físicas e deformidades permanentes ${ }^{(18-19,22,24,28,32,34)}$.

Conforme identificado nos estudos, as tecnologias educacionais e assistenciais foram utilizadas nos cuidados dos pacientes com hanseníase pelos seguintes profissionais da área da saúde: enfermeiros, fisioterapeutas, terapeuta ocupacional e assistentes sociais ${ }^{(26,28,34)}$. Esses cuidados foram realizados, em sua maioria, em nível de atenção secundária a saúde (18-19,23-24,26,28,30,32) $_{\text {. }}$

\section{Discussão}

As principais tecnologias em saúde para a promoção do autocuidado em pacientes com hanseníase identificadas nos estudos foram educacionais e assistenciais. Estas utilizaram diversas estratégias e/ou materiais para prevenção e redução das incapacidades e/ou deformidades físicas.

Nesta perspectiva, as tecnologias contemplam atos de caráter educativo em hanseníase, que devem ser horizontais e constituídas em um exemplar expressivo, edificante, dialógico, comunicativo e de atuação coletiva para o empoderamento do paciente na sua adesão às práticas de autocuidado e, consequentemente, para a prevenção das incapacidades ${ }^{(19)}$.

As estratégias educacionais, especialmente as oficinas terapêuticas, são atividades de encontro de vidas entre pessoas, que promovem a liberdade e a convivência dos diferentes. Trata-se de uma importante forma de tratamento, que busca a construção de desenvolturas corpóreas, consumação de práticas bem-sucedidas e atividades coletivas ${ }^{(35)}$. Estas apresentam-se com potencial para auxiliar na reabilitação psíquica de pacientes institucionalizados em decorrência da hanseníase ${ }^{(27)}$.

Ainda no contexto das tecnologias educativas, um estudo de intervenção educativa realizado em uma Unidade Básica de Saúde do Estado de Minas Gerais, apresentou a educação em saúde como uma importante estratégia para o autocuidado em pessoas com hanseníase. Essa, quando direcionada e aplicada, pode contribuir para um satisfatório acréscimo de conhecimento, favorecendo a prevenção de incapacidades físicas ${ }^{(36)}$.

É importante destacar que a própria educação em saúde é uma tecnologia. Suas ações promovem o conhecimento e capacitam os pacientes sobre as práticas de autocuidado. No entanto, essas ações precisam ser monitoradas e avaliadas pelos profissionais de saúde, para garantir que sejam desenvolvidas de forma correta e eficaz. Sendo assim, as tecnologias assistenciais de avaliação e acompanhamento são imprescindíveis para o controle e a prevenção de incapacidades.

As tecnologias educacionais utilizadas nos estudos permitiram o encontro com o outro, para discutir conjuntamente ações de promoção da saúde, que, em sua maioria, se mostraram efetivas na redução de deformidades e incapacidades físicas geradas pela hanseníase. Essas ações e tecnologias evidenciaram as nuances que as estratégias em educação em saúde devem assumir para estimular e promover a construção de saberes frente ao autocuidado das pessoas que convivem com hanseníase.

Percebe-se que as tecnologias educacionais para promoção do autocuidado em pessoas com hanseníase foram utilizadas com o intuito de 
incitar a conscientização sobre a gama de riscos existentes contra a integridade física e estimular a realização do autocuidado, com a transformação de atitudes. Além disso, estimularam o empoderamento e a autonomia, visando o reconhecimento de um problema e trabalhar para que ocorra a superação.

Em relação às mudanças na prevenção, controle e tratamento da hanseníase, diversas formas de tecnologias têm sido utilizadas no contexto da saúde e têm proporcionado resultados positivos, por meio de troca de experiências e busca de novas formas de cuidados. Essas mudanças foram identificadas nos estudos com grupos de apoio para pessoas com hanseníase que utilizou folhetos impressos e materiais didáticos sobre o autocuidado. Tais aspectos foram evidenciados em estudo ${ }^{(29)}$ no qual a maioria dos participantes (86\%) obteve redução das incapacidades físicas, além de apresentar satisfação em participar dos grupos.

A promoção das práticas do autocuidado tem, em sua raiz, a proposta de fomentar a autonomia do paciente no cuidado de si. Baseiam-se na troca de saberes e experiências, favorecendo o exercício da cidadania e do protagonismo em saúde ${ }^{(37)}$.

Neste estudo, emergiram também as Tecnologias Assistenciais, conhecidas como aparelhos que proporcionam a intercessão de processos de cuidar. São executadas por profissionais com o público alvo (pacientes/usuários) dos sistemas de saúde, nos três níveis de complexidade ${ }^{(9)}$.

Dentre as tecnologias apresentadas, a observação, a entrevista e o uso de instrumentos para avaliação do autocuidado apresentaram-se como estratégias-chave para a identificação de necessidades de saúde da população.

Outra tecnologia assistencial utilizada foi o Processo de Enfermagem (PE). Estes profissionais foram apontados nos artigos analisados como aqueles que mais estiveram vinculados às ações para promoção do autocuidado de pacientes com hanseníase.

A consulta de Enfermagem torna-se primordial na assistência ao portador de hanseníase, por possibilitar ao profissional estabelecer uma relação terapêutica com o paciente. Além disso, possibilita-lhe reconhecer as condições de vida e saúde que determinarão as demandas e os perfis dos processos que envolvem a saúde e o adoecimento dos usuários do serviço ${ }^{(38)}$.

O PE, aqui entendido como uma tecnologia assistencial, por se tratar de um instrumento metodológico que orienta o cuidado de enfermagem, contribui para a organização e promoção de estratégias de cuidado específicas para atender as demandas da clientela de forma individualizada ${ }^{(39)}$. No âmbito do autocuidado, o PE manifesta-se como uma metodologia de cuidar que proporciona a otimização da assistência, favorecendo a sistematização de ações de cuidados que viabilizem o alcance de resultados esperados ${ }^{(40)}$.

Os cuidados de Enfermagem voltados ao paciente com hanseníase devem estar balizados pelas necessidades e demandas de saúde evidenciadas pelos usuários dos serviços. Tendo em vista que, no presente estudo, a maioria dos participantes foi classificada como multibacilar, fica evidente a necessidade do uso das tecnologias educacionais, assistenciais e gerenciais para promoção do autocuidado/prevenção de incapacidades e, consequentemente, a melhora da qualidade de vida dessa população.

Como limitação do estudo pode-se apontar a ausência de estudos que abordem as tecnologias gerenciais voltadas para a promoção do autocuidado em pacientes com hanseníase. Nesse sentido, evidencia-se a necessidade de estudos que contemplem tal temática.

\section{Conclusão}

Os achados mostram que as tecnologias educativas e assistenciais são importantes para a prática de autocuidado em pacientes com hanseníase. Observou-se que o uso das tecnologias educacionais é relevante para a redução das incapacidades físicas ocasionadas pela hanseníase. No entanto, é imprescindível o desenvolvimento de tecnologias acessíveis aos pacientes, que auxiliem na prevenção da hanseníase, para que se possa romper com a cadeia de transmissão.

Quanto à tecnologia assistencial, possibilita ampliar o processo de trabalho do profissional 
12

Tecnologias em saúde para a promoção do autocuidado em pacientes com hanseníase: explorando evidências científicas

de saúde, manter o vínculo, a interação e possibilitar a construção compartilhada de alternativas que melhorem a qualidade de vida e saúde dessa população. Nesse sentido, é necessário que os profissionais de saúde, em especial o enfermeiro, incorpore as tecnologias em saúde, no ambiente de trabalho, por meio de formação continuada e do desenvolvimento de pesquisas que evidenciem e ressignifiquem as práticas de saúde. Além disso, articular as ações entre os diversos níveis de atenção à saúde, de modo a garantir um cuidado e um autocuidado de qualidade desenvolvido pela pessoa em tratamento da hanseníase.

Este estudo contribui para identificação dos principais tipos de tecnologias em saúde aplicadas às pessoas com hanseníase, com o propósito de intensificar esse uso para a promoção do autocuidado e prevenção de incapacidades físicas. Contribui ainda para ampliar as ações de enfermagem na prática de autocuidado, de modo a minimizar os danos causados pela doença.

\section{Colaborações:}

1 - concepção, projeto, análise e interpretação dos dados: Jeane Lima Cavalcante, Samyra Paula Lustoza Xavier, Jennifer Ferreira Figueiredo Cabral, Maria Corina Amaral Viana e Edilma Gomes Rocha Cavalcante;

2 - redação do artigo e revisão crítica relevante do conteúdo intelectual: Jeane Lima Cavalcante, Samyra Paula Lustoza Xavier, Jennifer Ferreira Figueiredo Cabral, Maria Corina Amaral Viana e Edilma Gomes Rocha Cavalcante;

3 - aprovação final da versão a ser publicada: Jeane Lima Cavalcante, Samyra Paula Lustoza Xavier, Jennifer Ferreira Figueiredo Cabral, Maria Corina Amaral Viana e Edilma Gomes Rocha Cavalcante.

\section{Referências}

1. Uchôa REMN, Brito KKG, Santana EMF, Soares VL, Silva MA. Perfil clínico e incapacidades físicas em pacientes com hanseníase. Rev Enferm UFPE on line. 2017;11(3):1464-72. DOI: 10.5205/ reuol.10263-91568-1-RV.1103sup201719
2. World Health Organization. Relevé épidémiologique hebdomadaire. Weekly epidemiol record [Internet]. 2016 Sep 2 [cited 2017 Mar 14];91(35):405-20. Available from: https://www. who.int/wer/2016/wer9135/fr/

3. Pescador MA, Sakae TM, Magajewsky FRL. Análise de tendência histórica da evolução da hanseníase em Santa Catarina no período de 2001-2015. Arq Catarin Med [Internet]. 2018 [cited 2018 Dec 3];47(1):141-58. Available from: http://www.acm. org.br/acm/seer/index.php/arquivos/article/ view/307/234

4. Brasil. Ministério da Saúde. Portaria no 3.125, de 7 de outubro de 2010. Aprova as diretrizes para vigilância, atenção e controle da hanseníase. Diário Oficial da República Federativa do Brasil, Brasília, 2010 out; Seção 1:55.

5. Souza MF, Vanderlei LCM, Frias PG. Avaliação da implantação do Programa de Controle da Hanseníase em Camaragibe, Pernambuco. Epidemiol Serv Saúde [online]. 2017;26(4):817-34. DOI: $10.5123 /$ S1679-49742017000400013

6. Zanardo TS, Santos SM, Oliveira VCC, Mota RM, Mendonça BOM, Nogueira DS, et al. Perfil epidemiológico dos pacientes com hanseníase na atenção básica de saúde de São Luís de Montes Belos, no período de 2008 a 2014. Rev Fac Montes Belos [Internet]. 2016 [cited 2018 Dec 3];9(2):77-141. Available from: http://revista.fmb. edu.br/index.php/fmb/article/view/226/203

7. Brasil. Ministério da Saúde. Secretaria de Vigilância em Saúde. Departamento de Vigilância Epidemiológica. Manual de Prevenção de incapacidades. Série A. Normas e Manuais Técnicos. Cadernos de prevenção e reabilitação em hanseníase. Brasília (DF); 2008 [cited 2018 Dec 3]. Available from: http://bvsms.saude.gov.br/bvs/ publicacoes/manual_prevencao_incapacidades. pdf

8. Nietsche EA, Backes VMS, Colomé CLM, Ceratti RN, Ferraz F. Tecnologias educacionais, assistenciais e gerenciais: uma reflexão a partir da concepção dos docentes de enfermagem. Rev Latino-Am Enfermagem. 2005;13(3):344-52. DOI: http://dx.doi.org/10.1590/ S0104-11692005000300009

9. Teixeira E. Tecnologias em enfermagem: produções e tendências para a educação em saúde com a comunidade. Rev Eletrôn Enferm. 2010;12(4):598-600. DOI: http://dx.doi. org/10.5216/ree.v12i4.12470 
Jeane Lima Cavalcante, Samyra Paula Lustoza Xavier,

10. Kessler M, Thumé E, Duro SMS, Tomasi E, Siqueira FCV, Silveira DS, et al. Ações educativas e de promoção da saúde em equipes do programa Nacional de Melhoria do Acesso e da Qualidade da Atenção Básica, Rio Grande do Sul, Brasil. Epidemiol Serv Saúde. 2018;27(2):e2017389. DOI: $10.5123 /$ S1679-49742018000200019

11. Leal DR, Cazarin G, Bezerra LCA, Albuquerque ACD, Felisberto E. Programa de Controle da Hanseníase: uma avaliação da implantação no nível distrital. Saúde em Debate. 2017;41(spe):209-28. DOI: https://doi. org/10.1590/0103-11042017S16

12. Whittemore R, Knafl K. The integrative review: updated methodology. J Adv Nurs. 2005;52(5):54653. DOI: $10.1111 /$ j.1365-2648.2005.03621.x

13. Mendes KDS, Silveira RCCP, Galvão CM. Revisão Integrativa: método de pesquisa para a incorporação de evidências na saúde e na enfermagem. Texto Contexto Enferm. 2008;17(4):758-64. DOI: http:// dx.doi.org/10.1590/S0104-07072008000400018

14. Galvão TF, Pansani TSA, Harrad D. Principais itens para relatar Revisões sistemáticas e Metaanálises: A recomendação PRISMA. Epidemiol Serv Saúde. 2015;24(2):335-42. DOI: 10.5123/ S1679-49742015000200017

15. Sampaio RF, Mancini MC. Estudos de revisão sistemática: um guia para síntese criteriosa da evidência científica. Rev bras fisioter. 2007;11(1):83-9. DOI: http://dx.doi.org/10.1590/ S1413-35552007000100013

16. Lima MCV, Barbosa FR, Santos DCM, Nascimento RD, D’Azevedo SSP. Práticas de autocuidado em hanseníase: face, mãos e pés. Rev Gaúcha Enferm. 2018;39:e20180045. DOI: https:// doi.org/10.1590/1983-1447.2018.20180045

17. Pryce J, Mableson HE, Choudhary R, Pandey BD, Aley D, Betts H, et al. Assessing the feasibility of integration of self-care for filarial lymphoedema into existing community leprosy self-help groups in Nepal. BMC public health. 2018;18(1):201. DOI: 10.1186/s12889-018-5099-0

18. D'Azevedo SSP, Freitas EN, Nascimento LO, Santos DCM, Nascimento RD. Percepção de pacientes com Hanseníase acerca dos Grupos de Autocuidado. Rev enferm UFPE on line. 2018;12(6):1633-9. DOI: https://doi.org/10.5205/19818963-v12i6a230855p1633-1639-2018

19. Morais JR, Furtado ÉZL. Grau de incapacidade física de pacientes com hanseníase. Rev Enferm
UFPE [Internet]. 2018;12(6):1625-32. DOI: https://doi. org/10.5205/1981-8963-v12i6a231049p1625-1632-2018

20. Moura EGS, Araújo APM, Silva MCR, Cardoso BA, Holanda MAS, Conceição AO, et al. Relação entre a Classificação Internacional de Funcionalidade, Incapacidade e Saúde (CIF) e a limitação de atividades e restrição à participação de indivíduos comhanseníase. CadSaúde Coletiva. 2017;25(3):35561. DOI: 10.1590/1414-462X201700030336

21. Maia FB, Teixeira ER, Silva GV, Gomes MK. The use of assistive technology to promote care of the self and social inclusion in patients with sequels of leprosy. PLoS Negl Trop Dis. 2016;10(4);e0004644. DOI: 10.1371/journal. pntd.0004644

22. Galan NGA, Beluci ML, Marciano LHSC, Prado RBR, Oliveira NGG, Bonini AG, et al. Avaliação da prática do autocuidado domiciliar em hanseníase. Hansen Int [Internet]. 2014 [cited 2018 Nov 16];39(2):27-35. Available from: file://C:/ Users/Jeane/Downloads/v39n2a04\%20(5).pdf

23. Batista TVG, Vieira CSCA, Paula MAB. A imagem corporal nas ações educativas em autocuidado para pessoas que tiveram hanseníase. Physis: Rev Saúde Coletiva. 2014;24(1):89-104. DOI: http:// dx.doi.org/10.1590/S0103-73312014000100006

24. Souza IA, Ayres JA, Meneguin S, Spagnolo RS. Autocuidado na percepção de pessoas com hanseníase sob a ótica da complexidade. Escola Anna Nery Rev Enferm. 2014;18(3):510-4. DOI: http://dx.doi.org/10.5935/1414-8145.20140072

25. de Vries HJ, de Groot R, van Brakel WH. Social participation of diabetes and ex-leprosy patients in the Netherlands and patient preference for combined self-care groups. Front Med. 2014 Aug;1:21. DOI:10.3389/fmed.2014.00021

26. Duarte LMCPS, Simpson CA, Silva TMS, Moura IBL, Isoldi DMR. Ações de autocuidado de pessoas com hanseníase. Rev Enferm UFPE on line. 2014;8(8):2816-22. DOI: 10.5205/ reuol.6081-52328-1-SM.0808201432

27. Leite SCC, Caldeira AP. Oficinas terapêuticas para a reabilitação psíquica de pacientes institucionalizados em decorrência da hanseníase. Ciênc Saúde Coletiva. 2015;20(Spe):1835-42. DOI: https://doi. org/10.1590/1413-81232015206.16412014

28. Pinheiro MGC, Silva SYB, Silva FS, Ataide CAV, Lima IB, Simpson CA. Conhecimento sobre prevenção de incapacidades em um grupo de autocuidado em hanseníase. Reme - Rev Min 
Enferm. 2014;18(4):895-906. Available from: DOI: $10.5935 / 1415-2762.20140066$

29. Deepak S, Hansine PE, Braccini C. Self-care groups of leprosy-affected people in Mozambique. Lepr Rev [Internet]. 2013 [cited 2018 Nov 16];84(4):283-91. Available from: https:// www.ncbi.nlm.nih.gov/pubmed/24745127

30. Palmeira IP, Ferreira MA. "O corpo que eu fui e o corpo que eu sou”: concepções de mulheres com alterações causadas pela hanseníase. Texto Contexto-Enferm. 2012 abr-jun;21(2):379-86. DOI: http://dx.doi.org/10.1590/S0104-07072012000200016

31. Cross H. The prevention of leprosy related disability as an integral component of the government health delivery programme in Myanmar. Lepr Rev [Internet]. 2011 [cited 2018 Nov 16];83(2):205-17. Available from: https://www.ncbi.nlm.nih.gov/ pubmed/22997697

32. Rodini FCB, Gonçalves $M$, Barros ARSB, Mazzer N, Elui VMC, Fonseca MCR. Prevenção de incapacidade na hanseníase com apoio em um manual de autocuidado para pacientes. Fisioter Pesq. 2010 Apr/Jun;17(2);157-66. DOI: http:// dx.doi.org/10.1590/S1809-29502010000200012

33. Sathia-Raj Y, Norman G, Richard J. Long term sustainability and efficacy of self-care education on knowledge and practice of wound prevention and management among leprosy patients. Indian J Lepr [Internet]. 2010 [cited 2018 Nov 16];82(2):7983. Available from: https://www.ncbi.nlm.nih.gov/ pubmed/21434510

34. Silva Júnior FJG, Ferreira RD, Araújo OD, Camêlo SMA, Nery IS. Assistência de enfermagem ao portador de Hanseníase: abordagem transcultural. Rev bras enferm. 2008:61(n spe):713-7. DOI: http://dx.doi.org/10.1590/S0034-71672008000700010
35. Ferreira KF, Carvalho VCS. Oficinas terapêuticas: caminhos de saberes. Rev Fac Ciênc Méd Sorocaba. 2018;20(2):82-5. DOI:10.23925/1984-4840.2018v20i2a5

36. Moreira AJ, Naves JM, Fernandes LFRM, Castro SS, Walsh IAP. Ação educativa sobre hanseníase na população usuária das unidades básicas de saúde de Uberaba-MG. Saúde Debate. 2014;38(101):234-43. DOI: $10.5935 / 0103-1104.20140021$

37. Silva MCD, Paz EPA. Educação em saúde no programa de controle da hanseníase: a vivência da equipe multiprofissional. Esc Anna Nery Rev Enferm. 2010;14(2):223-9. DOI: http://dx.doi.org/ 10.1590/S1414-81452010000200003

38. Lima DAQ, Cassemir AVS, Mendes RS, Branco CSN, Pamplona YAP. Consulta de enfermagem ao portador de hanseníase. Rev Enferm Contemp. 2015 jul/dez;4(2):199-208. DOI: 2317-3378rec.v4i2.387

39. Trindade LR, Ferreira AM, Silveira A, Rocha EN. Processo de enfermagem: desafios e estratégias para sua implementação sob a ótica de enfermeiros. Saúde (Santa Maria). 2016;42(1);75-82. DOI: http:// dx.doi.org/10.5902/2236583419805

40. Bezerra MLR, Nunes SFL, Jesus CAC. Diagnósticos de enfermagem com foco no problema para indivíduos acometidos pela hanseníase. Rev Enferm Atual [Internet]. 2019 [cited 2019 May 15];88(26):1-7. Available from: http://revistaenfermagematual. com.br/index.php/revista/article/view/381.

Recebido: 30 de agosto de 2019 Aprovado: 18 de novembro de 2019 Publicado: 11 de março de 2020

A Revista Baiana de Enfermagem utiliza a Licença Creative Commons - Atribuição-NãoComercial 4.0 Internacional. https://creativecommons.org/licenses/by-nc/4.0/

Este artigo é de acesso aberto distribuído sob os termos da Licença Creative Commons (CC BY-NC). Esta licença permite que outros remixem, adaptem e criem a partir do seu trabalho para fins não comerciais. Embora os novos trabalhos tenham de lhe atribuir o devido crédito e não possam ser usados para fins comerciais, os usuários não têm de licenciar esses trabalhos derivados sob os mesmos termos. 